LAVAL-PHY-96-17

ALBERTA-THY-39-96

\title{
Scalar Leptoquark Pair Production at the CERN LHC: Signal and Backgrounds
}

\author{
B. Dion, L. Marleau, G. Simon \\ Département de Physique, Université Laval \\ Québec QC Canada, G1K $7 P_{4}$ \\ M. de Montigny \\ Faculté Saint-Jean, University of Alberta \\ Edmonton AB Canada, T6C $4 G 9$
}

(1996)

\begin{abstract}
We present the results of an analysis for the pair production of scalar leptoquarks at the CERN Large Hadron Collider (LHC) with $\sqrt{s}=14 \mathrm{TeV}$ and $\mathcal{L}=10 \mathrm{fb}^{-1}$ which includes the dominant sources of Standard Model background associated to this process: $t \bar{t}, Z Z, W Z$ and $Z^{*} j j$ production. The $t \bar{t}$ process provides the main source of background. We consider leptoquarks introduced in the framework of a superstring-inspired $E_{6}$ model. The leptoquark production is found to be dominant in all regions of parameter space for leptoquark masses below $750 \mathrm{GeV}$. We establish the discovery reach of the leptoquarks at $750 \mathrm{GeV}(1 \mathrm{TeV})$ for a branching ratio of $B(L Q \rightarrow e q)=0.5$ $(B=1)$.
\end{abstract}

PACS numbers: 11.25.Mj, 13.85.Qk, 14.80.-j. 


\section{INTRODUCTION}

H1 [1] and ZEUS [2] experiments have recently reported an excess of deep inelastic neutral current events in the range $Q^{2} \geq 15000 \mathrm{GeV}^{2}$. This has prompted several theoretical and phenomenological analyses [3] seeking a proper interpretation. One such interpretation for these events suggests single scalar leptoquark production in the $e^{+} q$ or $e^{+} \bar{q}$ channels. Although the statistics for these high- $Q^{2}$ events remain quite low for now (12 events) and no confirmation can be drawn until further measurements are performed, it is nonetheless interesting to look at the discovery possibilities of scalar leptoquarks at existing or future hadron colliders.

Leptoquarks are known to occur in various extensions of the SM, such as composite [4], GUT [5] and SUSY [6] models, as exotic particles which carry both color and lepton quantum numbers. In general, they are either scalar or vector particles, with mass and coupling constant to the standard fermions left as unknown parameters. Some experimental contraints have been set on these parameters quite recently [0, 8, 9, 10, 11].

Leptoquarks can be directly produced in $e p$ colliders but their pair production at hadron colliders still has a clear advantage over any other method: it is almost insensitive to the magnitude of the Yukawa coupling which is unknown. Previous searches performed at the proton-antiproton collider Tevatron (Fermilab) have excluded scalar leptoquarks with masses below $175 \mathrm{GeV}$ and $147 \mathrm{GeV}$ for branching ratios of the leptoquarks to the electron equal to 1 and 0.5 respectively [7]. For the second generation, CDF sets limits and obtains 180 $\mathrm{GeV}(140 \mathrm{GeV})$ for $B=1(0.5)$ [ 8 . Similarly, a limit of $99 \mathrm{GeV}$ for $B=1$ was obtained by CDF for third generation leptoquarks [9]. Some searches have also been performed at LEP 10] and previous HERA runs have also contributed to set limits [11]. A large machine like the LHC (with $\sqrt{s}=14 \mathrm{TeV}$ and $\mathcal{L}=10 \mathrm{fb}^{-1}$ ) should improve considerably such discovery limits.

The cross sections for the pair production of scalar leptoquarks at hadron colliders can be found in the literature [12,13,14,15]. However, a comprehensive study of the various QCD and electroweak backgrounds which accompany leptoquark processes has been lacking up until recently [16]. Indeed, it is not trivial otherwise to estimate to which extent the leptoquark signal will "survive" the QCD production of heavy fermions, or jets produced along with the vector bosons $W$ and $Z$, etc. Here, we shall consider events where both leptoquarks decay into an electron plus quark, implying a 2 jets $+e^{+} e^{-}$signature.

The purpose of this paper is to investigate the ability of the CERN Large Hadron Collider (by using the design of the ATLAS and CMS experiments [17, 18]) to unravel the presence of scalar leptoquarks and to examine to which extent the leptoquark signal can be distinguished from Standard Model processes. We implement leptoquark data and related cross sections in the ISAJET event generator and use the ISZRUN package contained in the Zebra version to perform our selection cuts.

We consider the scalar leptoquarks contained in the supersymmetric grand unified $E_{6}$ model (the low-energy limit of an $E_{8} \otimes E_{8}$ heterotic string theory [19]). In the $E_{6}$ model, each matter supermultiplet lies in the fundamental $\mathbf{2 7}$ representation, which contains, in addition to the usual quarks and leptons (and their superpartners), new particles such as two fiveplets $(D, H)$ and $(\bar{D}, \bar{H})$ and an $S U(5)$ superfield singlet $N$. We focus on the superfields $D$ and $\bar{D}$ which are two $S U(3)$ triplets and $S U(2)$ singlets with electric charges $-1 / 3$ and 
$+1 / 3$, respectively. Depending on the charge assignment chosen for the superfields, $D$ and $\bar{D}$ can be taken to possess baryonic number $\pm 1 / 3$ and leptonic number \pm 1 . The scalar superpartners of these superfields are the object of the present study. We thus consider scalar leptoquarks with $Q=-1 / 3$. We restrict our study to the first generation of fermions. The Yukawa interactions take the form:

$$
\mathcal{L}_{Y}=\lambda_{L} \tilde{D}^{c *}\left(e_{L} u_{L}+\nu_{L} d_{L}\right)+\lambda_{R} \tilde{D} e_{L}^{c} u_{L}^{c}+\text { h.c. }
$$

where $c$ denotes the charge conjugate state, and $\tilde{D}$ is the scalar superpartner of $D$. In this model, the $\lambda$ are independent and arbitrary but we choose them to be equal to the electromagnetic charge, following [20]. It is important to note that leptoquarks also interact strongly. As we shall see, these interactions are mainly responsible for their production in pairs.

In the following Section, we present the details of our simulation and the selection cuts that we have chosen. Next, we elaborate on the expected signature of the leptoquark sig-

nal and of the principal sources of background: Drell-Yan and $t \bar{t}$ production. Finally, we summarize our results and conclude in Section V.

\section{EVENT SIMULATION}

\section{A. Detector and calorimeter}

We use the toy calorimer simulation package ISZRUN contained in the Zebra version of ISAJET [21] to simulate the experimental conditions at the LHC, with the ATLAS and CMS detectors in mind:

- cell size: $\triangle \eta \times \triangle \phi=0.05 \times 0.05$,

- pseudorapidity range: $-5<\eta<5$,

- hadronic energy resolution: $50 \% / \sqrt{E} \oplus 0.03$ for $-3<\eta<3$,

$$
100 \% / \sqrt{E} \oplus 0.07 \text { for } 3<|\eta|<5 \text {, }
$$

- electromagnetic energy resolution: $10 \% / \sqrt{E} \oplus 0.01$.

\section{B. Kinematic cuts}

For the purposes of this work, hadronic showers are regarded as jets when they

- lie within a cone of radius $R=\sqrt{(\Delta \eta)^{2}+(\Delta \phi)^{2}}=0.7$,

- possess a transverse energy $E_{T}>25 \mathrm{GeV}$,

- have a pseudorapidity $\left|\eta_{j}\right| \leq 3$.

Similarly, electrons are considered isolated if they 
- are separated from any jet by $R \geq 0.3$,

- have a transverse momentum $p_{T}>25 \mathrm{GeV}$,

- have a pseudorapidity $\left|\eta_{l}\right| \leq 2.5$.

Our calculations are performed using the PDFLIB distribution functions of Morfin and Tung (M-T B2) with $\Lambda=191 \mathrm{MeV}$ [22,23]. The choice of the distribution functions affects only slightly the cross section. The calculations were repeated with more recent distribution functions, namely CTEQ3M [23]. For leptoquark masses below $400 \mathrm{GeV}$, the results remain practically unchanged; for higher masses, the cross section is enhanced by at most $5 \%$.

\section{LEPTOQUARK SIGNAL AND BACKGROUNDS}

Here, we consider first-generation leptoquarks, which can decay into either an $u$-quark and an electron, or into a $d$-quark and a $\nu_{e}$. For the purposes of our calculations, we consider the case in which both occur with equal probability $(B=0.5)$. We also assume a Yukawa coupling of electromagnetic strength $\alpha_{Y}=\alpha_{e m}$ (in fact, it is a generic feature of string-inspired models that the non-zero Yukawa coupling is of the same order as the gauge coupling [24]). In fact, the Yukawa coupling has only a very small impact on the pair production cross section.

\section{A. Leptoquark signal}

We analyze the pair production of scalar leptoquarks which arise from two subprocesses: (1) quark-antiquark annihilation $\left(u_{R}+u_{L}^{c} \rightarrow \tilde{D}+\tilde{D}^{*}\right.$ and $\left.u_{L}+u_{R}^{c} \rightarrow \tilde{D}^{c *}+\tilde{D}^{c}\right)$, and (2) gluon fusion $\left(g+g \rightarrow \tilde{D}+\tilde{D}^{*}\right.$ and $\left.g+g \rightarrow \tilde{D}^{c *}+\tilde{D}^{c}\right)$ (see Fig. 1). Whereas the first subprocess occurs in the $s$-channel (through the exchange of a virtual gluon) and in the $t$-channel (virtual electron), subprocess (2) arises via color gauge interactions from the trilinear term $g D D$ in the $s$-channel (through the exchange of a gluon) and in the $t$ - and $u$-channels (exchange of virtual scalar leptoquarks), and from the quartic term $g g D D$ in which two gluons annihilate to produce a pair of leptoquarks.

In our calculations, we omitted the soft-gluon correction $K$-factors $13 \pi, K_{g g}=1+2 \alpha_{s} \pi / 3$ and $K_{q \bar{q}}=1-\alpha_{s} \pi / 6$, for gluon fusion and quark-antiquark annihilation respectively. Previous studies suggest that the gluon fusion subprocess will dominate at the LHC energies. Thus, we can expect a cross section enhancement factor ranging from 1.22 to 1.19 for leptoquark masses of $200 \mathrm{GeV}$ up to $1 \mathrm{TeV}$ (assuming $\mu=M_{L Q}$ which is the choice of scale used throughout these calculations). Recently, Krämer et al. [15] have carried out a complete NLO calculation of scalar leptoquark pair production; their results are expressed in the form of an overall $K$-factor which essentially reproduces the features of the soft-gluon $K$-factor approach with $\mu=M_{L Q}$.

Leptoquark pair production can lead to three distinct signals:

(a) 2 jets $+e^{+} e^{-}$,

(b) 2 jets $+\not p_{T}$, 


$$
\text { (c) } 2 \text { jets }+e^{ \pm}+\not p_{T} \text {. }
$$

The most striking of these signals is expected to be (a). In fact, signals (b) and (c) are more cumbersome because many SM ( $W W, W Z, Z Z, Z g g$ and $Z g q$ production) and SUSY processes have the same signatures. We therefore restrict ourselves to 2 jets $+e^{+} e^{-}$. The background which comes mainly from $t \bar{t}$ can be considerably reduced by requiring a cut on the transverse energy of both the jets and the leptons. Here we shall impose the same $E_{T}$ cut on the leptons and the jets.

\section{B. SM Backgrounds}

The most probable sources of background as identified by Refs. [12] are (1) $t \bar{t},(2) Z^{*} j j$, (3) $Z Z$ and $W Z$ production. However, our calculations have shown processes 2 and 3 (with an invariant mass cut on the lepton pair: $81 \mathrm{GeV} \leq M_{e^{+} e^{-}} \leq 101 \mathrm{GeV}$ ) to be negligible compared to (1). Therefore, we will restrict ourselves to $t \bar{t}$ (see Fig. 22.) where the top is decaying into a $b$ quark, an electron and a $\nu_{e}$. The presence of neutrinos implies in general a missing transverse momentum $\not p_{T}$. In this case, it is natural to expect the available transverse energy of the electron to be smaller on average than that involved in the leptoquark process. Our calculations were made using $M_{t}=175 \mathrm{GeV}$.

\section{LEPTOQUARK DISCOVERY REACH AT THE CERN LHC}

Fig. 3 shows the total cross section for 2 jets $+e^{+} e^{-}$as a function of the mass for a transverse energy cut $E_{T}=200 \mathrm{GeV}$. The leptoquark signal (solid line) is plotted against the leptoquark mass whereas the $t \bar{t}$ background (dashed line) is evaluated at $M_{t}=175 \mathrm{GeV}$. The $5 \sigma$ statistical significance is achieved for leptoquark masses up to $750 \mathrm{GeV}$. This limit also corresponds to 10 leptoquark events considering a luminosity of $10 \mathrm{fb}^{-1}$. Thus, we find a discovery reach of $750 \mathrm{GeV}$ for leptoquarks that decay into electrons with $B=0.5$. We can also evaluate the discovery limit for leptoquarks that decay with $B=1$ by recalling that the cross section for the production of 2 jets $+e^{+} e^{-}$is four times larger in this case. This leads to a discovery reach of $1 \mathrm{TeV}$. Our discovery limits are somewhat lower than those recently obtained in Ref. [14]. This is expected since the cuts that we have applied to suppress the background have also reduced the signal cross section.

One of the features of the leptoquark production process is the strong correlation between the jet and the electron emerging from the same leptoquark. In order to illustrate this fact, we look at the invariant mass distribution of the lepton-jet pair $\left(M_{e j}\right)$ of leptoquark pairs and the $t \bar{t}$ background. The reconstruction of leptoquarks from lepton-jet pairs raise the problem of conveniently pairing each lepton with the right jet. A method consist of associating the lowest-energy lepton with the highest-energy jet, but it did not turn out to be the most efficient procedure here. Instead, pairing the electrons and the jets using event topology (i.e. matching an electron with its nearest-neighbor jet) gave much better results. At the LHC, the pairs of leptoquarks in the mass range under study $\left(M_{L Q} \ll \sqrt{s}\right)$ are produced with very high kinetic energy in opposite directions which explains why the decay products of each leptoquark appear predominantly in opposite hemispheres. We present our results 
for the invariant mass distribution of the lepton-jet pair $\left(M_{e j}\right)$ in Figs. 5 for $E_{T}$ cuts of $100 \mathrm{GeV}$ and $200 \mathrm{GeV}$ respectively. The solid lines correspond to the leptoquark signal with (a) $M_{L Q}=200 \mathrm{GeV}$, (b) $M_{L Q}=500 \mathrm{GeV}$ and (c) $M_{L Q}=750 \mathrm{GeV}$. The dashed lines correspond to $t \bar{t}$ background. The lepton-jet correlation is quite evident when looking at the peaks in the $M_{e j}$ distribution. In comparison, the background does not exhibit any such peaks as can be expected from the presence of a missing $\not p_{T}$. The signal-to-background ratio is optimal for an $E_{T}$ cut of $200 \mathrm{GeV}$ (Fig. 5).

In order to emphasize the importance of the signal-to-background ratio near the peak in the $M_{e j}$ distribution, we display in Fig. 6 the partial cross section integrated over a bin

of width $\Delta M_{e j}=100 \mathrm{GeV}$ around $M_{e j}=M_{L Q}$ as a function of the invariant mass of the electron-jet pair for $E_{T}=200 \mathrm{GeV}$. The results are presented for a large set of intermediate values of $M_{e j}=M_{L Q}$ within the range $100 \mathrm{GeV}<M_{L Q}<1 \mathrm{TeV}$. The leptoquark signal (solid line) exhibits a smooth logarithmic behavior while the $t \bar{t}$ background shows some irregular fluctuations around an approximatively constant value. Note that these fluctuations can be misleading on a logarithmic plot as they turn out to be rather small in magnitude. Comparing with Fig. 3, we find that the signal-to-background ratio is increased by one order of magnitude in Fig. 6. The $5 \sigma$ statistical significance is achieved for leptoquark masses up to $1 \mathrm{TeV}$.

In conclusion, we have presented the results of a complete analysis of the first-generation scalar leptoquark pair production within the context of an $E_{6}$ model. We have also calculated the importance of the various Standard Model backgrounds which have the same signature. The leptoquark signal was found to be dominant over the $t \bar{t}$ background for leptoquark masses up to $750 \mathrm{GeV}$. We have evaluated our leptoquark discovery limit for the optimal case $E_{T}=200 \mathrm{GeV}$. We found a leptoquark discovery reach of $750 \mathrm{GeV}$ (1 TeV) for a branching ratio of $B(L Q \rightarrow e q)=0.5(B=1)$.

\section{ACKNOWLEDGMENTS}

We are indebted to Pr. J. Pinfold for useful comments. The authors would like to thank Pr. Frank Paige for answering numerous questions during the installation of ISAJET. This research was supported by the Central Research Fund of the University of Alberta, contract no CRF-GEN 81-53428, the Natural Sciences and Engineering Research Council of Canada, and by the Fonds pour la Formation de Chercheurs et l'Aide à la Recherche du Québec. 


\section{REFERENCES}

[1] C. Adloff et al., H1 collaboration, Z. Phys C74 (1997) 191.

[2] J. Breitweg et al., ZEUS collaboration, Z. Phys. C74 (1997) 207.

[3] J. Blümlein, DESY-97-105 and hep-ph/9706362; D. Choudhury and S. Raychaudhuri, hep-ph/9702392; T. Kuo and T. Lee, hep-ph/9703255; G. Altarelli et al., hepph/9703276; H. Dreiner and P. Morawitz, hep-ph/9703279; M. A. Doncheski and S. Godfrey, hep-ph/9703285; J. Blümlein, DESY-97-032 and hep-ph/9703287; J. Kalinowski et al.., hep-ph/9703288 and Z. Phys. C in print; K. Babu et al., hep-ph/9703299; V. Barger et al., hep-ph/9703311; M. Suzuki, hep-ph/9703316; M. Drees, hep-ph/9703332; J. Hewett and T. Rizzo, hep-ph/9703337; G. K. Leontaris and J.D. Vergados, hepph/9703338; M. C. Garcia and S. D. Novaes, hep-ph/9703346; D. Choudhury and S. Raychaudhuri, hep-ph/9703369; C. Papadopoulos, hep-ph/9703372; N. Di Bartolomeo and M. Fabbrichesi, hep-ph/9703375; A. Nelson, hep-ph/9703379; J. Kalinowski et al., hep-ph/9703436; B. Arbuzov, hep-ph/9703460; C. Friberg et al., hep-ph/9704214; T. Kon and T. Kobayashi, hep-ph/9704221; S. Jadach et al., hep-ph/9704241; A. White, hep-ph/9704248; R. Barbieri et al., hep-ph/9704275; I. Montvay, hep-ph/9704280; W. Buchmüller and D. Wyler, hep-ph/9704317; K. Akama et al., hep-ph/9704327; S. King and G. Leontaris, hep-ph/9704336; S. Kuhlmann et al., hep-ph/9704338; G. Giudice and R. Rattazzi, hep-ph/9704339; A. Belyaev and A. Gladyshev, hep-ph/9704343; J. Elwood and A. E. Faraggi, hep-ph/9704363; S. Godfrey, hep-ph/9704380; M. Chizov, hepph/9704409; B. Dutta, R. N. Mohapatra and S. Nandi, hep-ph/9704428; M. Heyssler and W. Stirling, hep-ph/9705229; N. Deshpande, B. Dutta and X-G. He, hep-ph/9705236; G. Altarelli, G. F. Giudice and M. L. Mangano, hep-ph/9705287; S. Barshay and G. Keyerhoff, hep-ph/9705303; D. Roy, hep-ph/9705370; K. Babu, C. Kolda and J. March-Russel, hep-ph/9705399:hep-ph/9705414; J. Ellis, S. Lola and K. Sridhar, hep-ph/9705416; A. Deandrea, hep-ph/9705435.

[4] L. Abbott and E. Fahri, Phys. Lett. B101 (1981) 69; Nucl. Phys. B189 (1981) 547; B. Schrempp and F. Schrempp, Phys. Lett. B153 (1985) 101 and references therein; J. Wudka, Phys. Lett. B167 (1985) 337.

[5] H. Georgi and S. L. Glashow, Phys. Rev. Lett. 32 (1974) 438; J. C. Pati and A. Salam, Phys. Rev. D10 (1974) 275; Phys. Rev. Lett. 31 (1973) 661; S. Dimopoulos and L. Susskind, Nucl. Phys. B155 (1979) 237; S. Dimopoulos, Nucl. Phys. B168 (1980) 69; P. Langacker, Phys. Rep. 72 (1981) 185; G. Senjanović and A. Šokorac, Z. Phys. C20 (1983) 255; R. J. Cashmore et al., Phys. Rep. 122 (1985) 275.

[6] E. Eichten, K. Lane and M.E. Peskin, Phys. Rev. Lett. 50 (1983) 811; J. L. Hewett and T. G. Rizzo, Phys. Rep. 183 (1989) 193; S. Dimopoulos, Nucl. Phys. B168 (1981) 69; E. Farhi and L. Susskind, Phys. Rev. D20 (1979) 3404; Phys. Rep. 74 (1981) 277; V. D. Angelopoulos et al., Nucl. Phys. B292 (1987) 59; J. F. Gunion and E. Ma, Phys. Lett. B195 (1987) 257; R. W. Robinett, Phys. Rev. D37 (1988) 1321; J. A. Grifols and S. Peris, Phys. Lett. B201 (1988) 287.

[7] J. Wightman et al., D0 Collaboration, Proceedings, Les Rencontres de la Vallee d'Aoste, 1997; J. Hobbs et al., D0 Collaboration, Proceedings, XXXII Rencontres de Moriond, 1997.

[8] K. Maeshima (CDF Collaboration), November 1996, Fermilab-Conf-96/413-E.

[9] F. Abe et al. (CDF Collaboration), December 1996, Fermilab-Pub-96/450-E. 
[10] G. Alexander et al. (OPAL Collaboration), Phys. Lett. B275 (1992) 123; Phys. Lett. B263 (1991) 123; D. Decamp et al. (ALEPH Collaboration), Phys. Rep. 216 (1992) 253; B. Adeva et al. (L3 Collaboration), Phys. Lett. B261 (1991) 169; O. Adriani et al. (L3 Collaboration), Phys. Rep. 236 (1993) 1; P. Abreu et al. (DELPHI Collaboration), Phys. Lett. B275 (1992) 222; Phys. Lett. B316 (1993) 620.

[11] I. Abt et al. (H1 Collaboration), Nucl. Phys. B396 (1993) 3; M. Derrick et al. (ZEUS Collaboration), Phys. Lett. B306 (1993) 173; T Ahmed et al. (H1 Collaboration), Z. Phys. C64 (1994) 545; S. Aid et al. (H1 Collaboration), Phys. Lett. B353 (1995) 578; Phys. Lett B369 (1996) 173.

[12] J. Grifols and A. Méndez, Phys. Rev. D26 (1982) 324; P. R. Harrison and C. H. Llewellyn Smith, Nucl. Phys. B213 (1983) 223 ; Erratum: Nucl. Phys. B223 (1983) 524; I. Antoniadis, L. Baulieu and F. Delduc, Z. Phys. C23 (1984) 119; E. Eichten, I. Hinchliffe, K. Lane and C. Quigg, Rev. Mod. Phys. 56 (1984) 579; G. Altarelli and R. Rückl, Phys. Lett. B144 (1984) 126; S. Dawson, E. Eichten and C. Quigg, Phys. Rev. D31 (1985) 1581; G. V. Borisov, Y. F. Pirogov and K. R. Rudakov, Z. Phys. C36 (1987) 217 J. L. Hewett and S. Pakvasa, Phys. Rev. D37 (1988) 3165; O. J. P. Éboli and A. Olinto, Phys. Rev. D38 (1988) 3461; T. G. Rizzo, SLAC-PUB-96-7284 and hep-ph/9609267.

[13] M. de Montigny, L. Marleau and G. Simon, Phys. Rev. D52 (1995) 533; M. de Montigny and L. Marleau, Phys. Rev. D40 (1989) 3616.

[14] J. Blümlein, E. Boos and A. Kryukov, DESY 96-174 and hep-ph/9610408.

[15] M. Krämer, T. Plehn, M. Spira and P.M. Zerwas, DESY-97-063 and hep-ph/9704322.

[16] B. Dion, L. Marleau and G. Simon, LAVAL-PHY-96-16 and hep-ph/9610397.

[17] W.W. Armstrong et al., ATLAS Technical Proposal, CERN/LHCC/94-43 (1994).

[18] G.L. Bayatian et al., CMS Technical Proposal, CERN/LHCC/94-38 (1994).

[19] M. Green and J. Schwarz, Phys. Lett. B149 (1984) 117; E. Witten, Nucl. Phys. B258 (1985) 75; D. Gross, J. Harvey, E. Martinec and R. Rohm, Phys. Rev. Lett. 54 (1985) 502; Nucl. Phys. B256 (1985) 253; Nucl. Phys. B267 (1986) 75; M. B. Green, J. H. Schwarz and E. Witten, "Superstring Theory", Cambridge University Press, New York (1987).

[20] J. L. Hewett and T. G. Rizzo, Phys. Rev. D36, (1987) 3367.

[21] F. Paige and S. Protopopescu, "Supercollider Physics", World Scientific (1986); H. Baer, F. Paige, S. Protopopescu and X. Tata, "Proceedings of the Workshop on Physics at Current Accelerators and Supercolliders", Argonne National Laboratory (1993).

[22] J. G. Morfin and W. K. Tung, Z. Phys. C52 (1991) 13.

[23] H. Plothow-Besh, Comput. Phys. Commun. 75 (1993) 396.

[24] E. Witten, Phys. Lett. B155 (1985) 151. 


\section{FIGURES}

FIG. 1. Feynman diagrams for leptoquark pair production via ((a), (b)) $q \bar{q}$ annihilation and ((c), (d), (e), (f)) gluon fusion.

FIG. 2. Feynman diagram for $t \bar{t}$ production.

FIG. 3. Integrated cross section for the production of 2 jets $+e^{+} e^{-}$as a function of the leptoquark mass for $E_{T}=200 \mathrm{GeV}$. The full line corresponds to the leptoquark signal versus the leptoquark mass $\left(M_{L Q}\right)$ and the dashed line to $t \bar{t}$ background for $M_{t}=175 \mathrm{GeV}$.

FIG. 4. Distribution of the invariant mass of the lepton-jet pair $\left(M_{e j}\right)$ for the production of 2 jets $+e^{+} e^{-}$for $E_{T}=100 \mathrm{GeV}$. The solid lines correspond to the leptoquark signal with (a) $M_{L Q}=200 \mathrm{GeV}$, (b) $M_{L Q}=500 \mathrm{GeV}$ and (c) $M_{L Q}=750 \mathrm{GeV}$. The dashed lines correspond to $t \bar{t}$ background.

FIG. 5. Same as figure 4 but for $E_{T}=200 \mathrm{GeV}$.

FIG. 6. Partial cross section within a bin of width $\Delta M_{e j}=100 \mathrm{GeV}$ around $M_{e j}=M_{L Q}$ as a function of the invariant mass of the electron-jet pair for $E_{T}=200 \mathrm{GeV}$. The full line corresponds to the leptoquark signal and the dashed line to $t \bar{t}$ background for $M_{t}=175 \mathrm{GeV}$. 


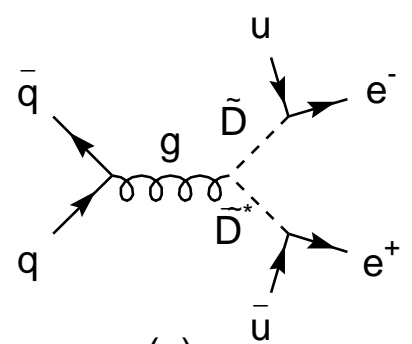

(a)

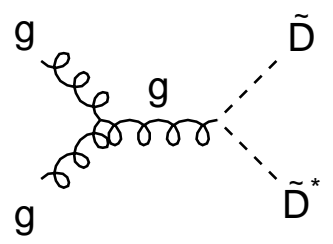

(c)

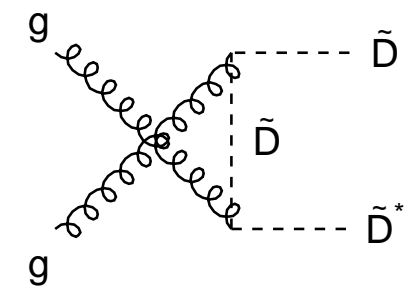

(e)

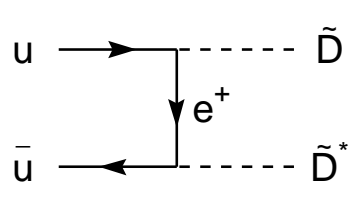

(b)

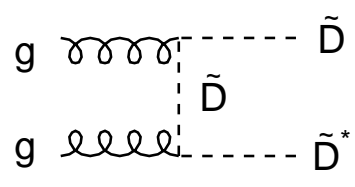

(d)

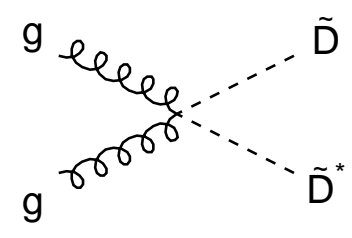

(f)

Figure 1 


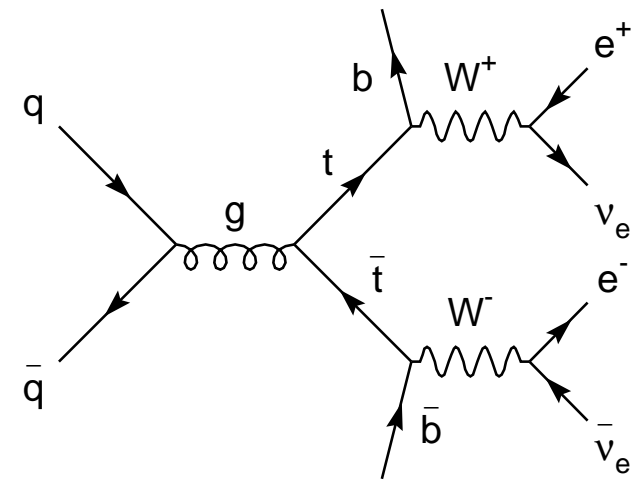

Figure 2 
Figure 3

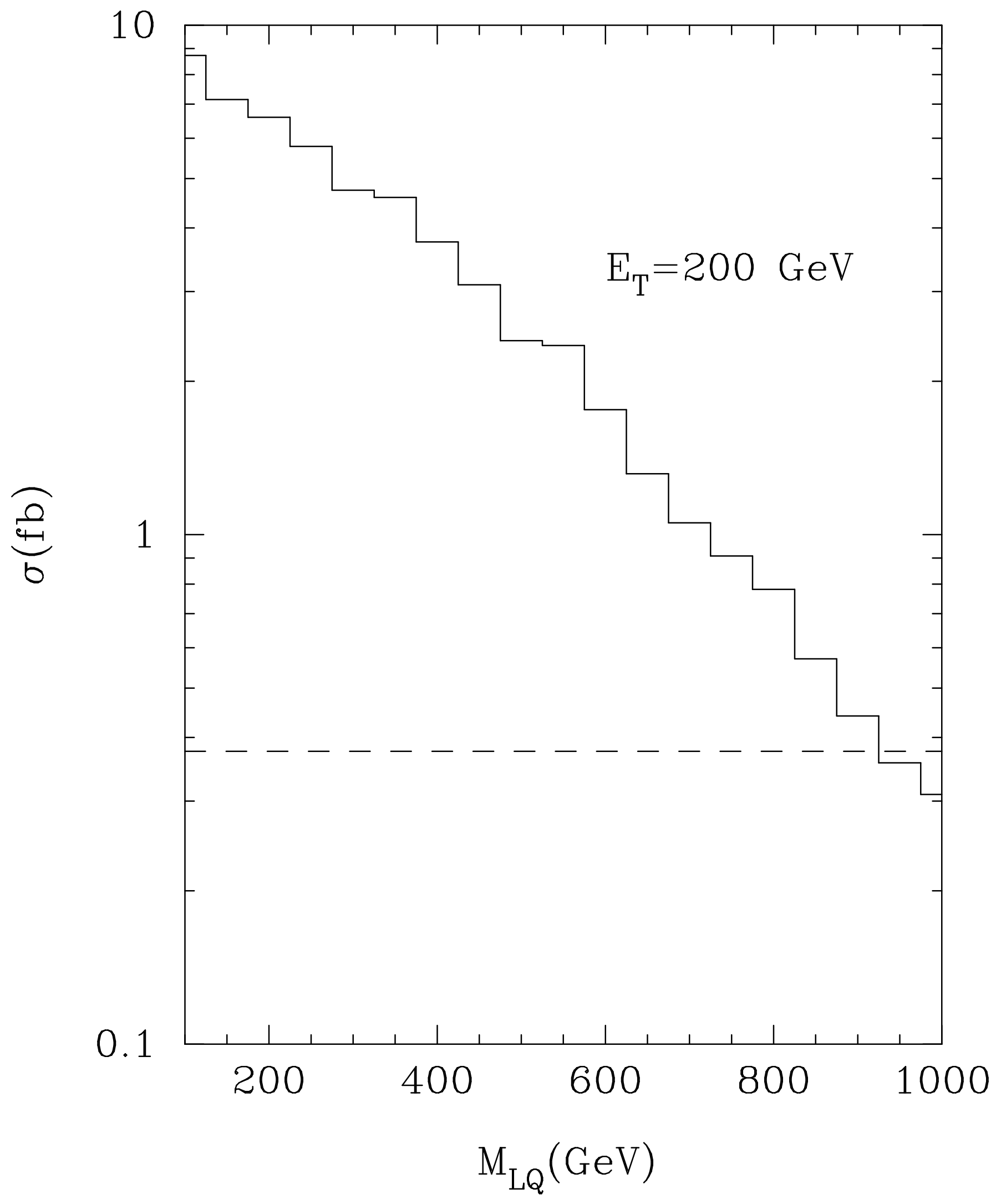


Figure $4 a$

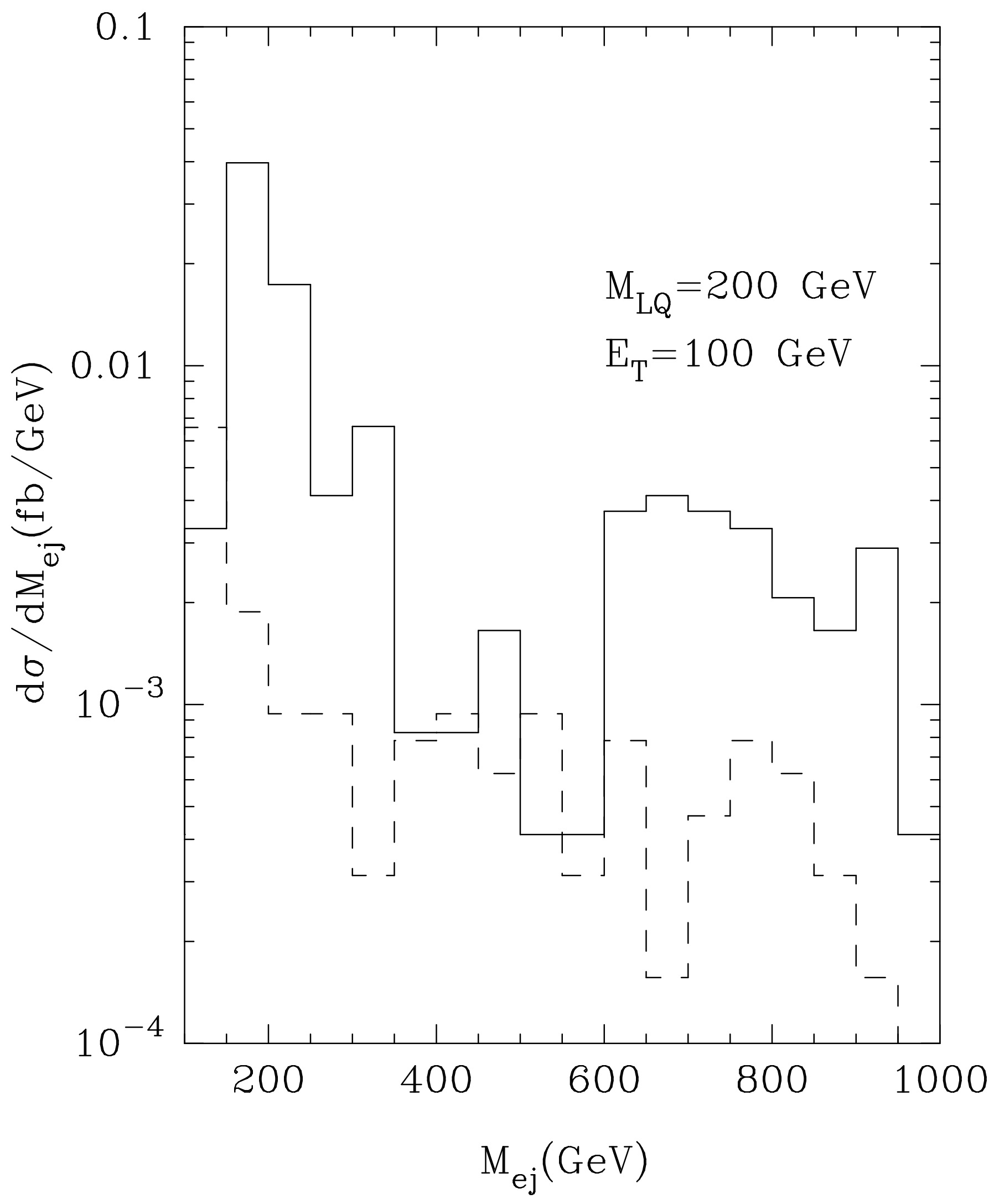


Figure 4b

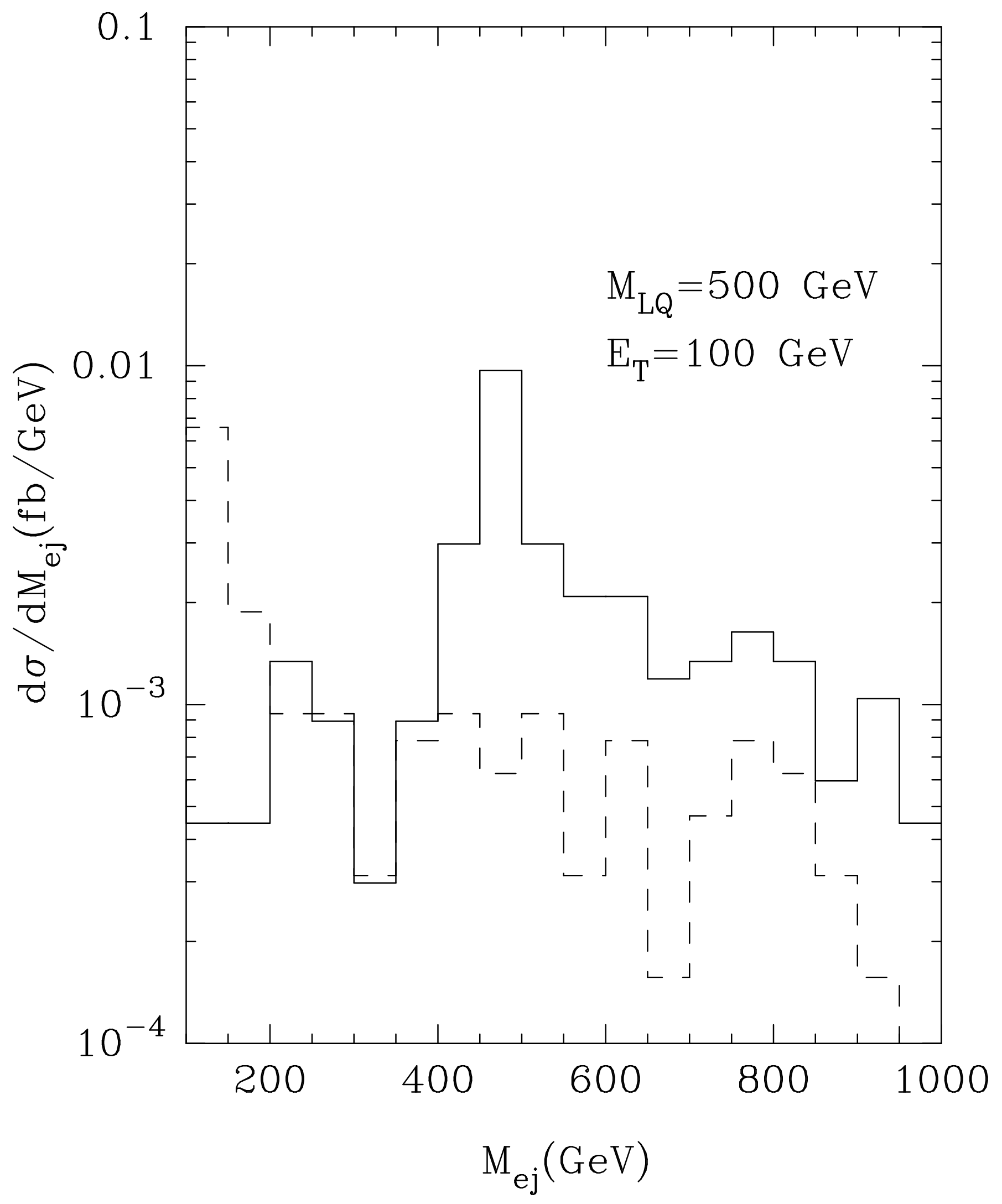


Figure 4c

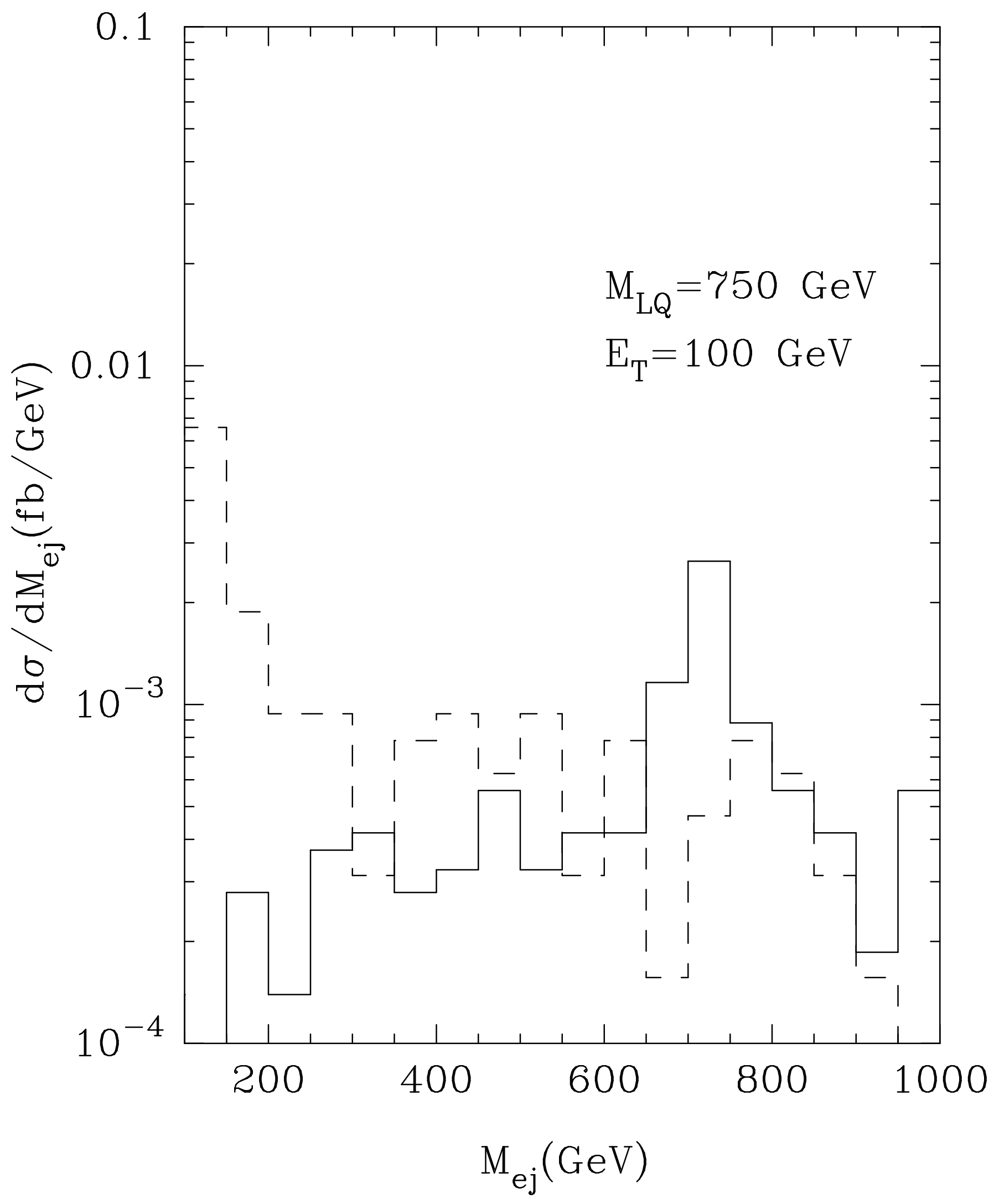


Figure $5 a$

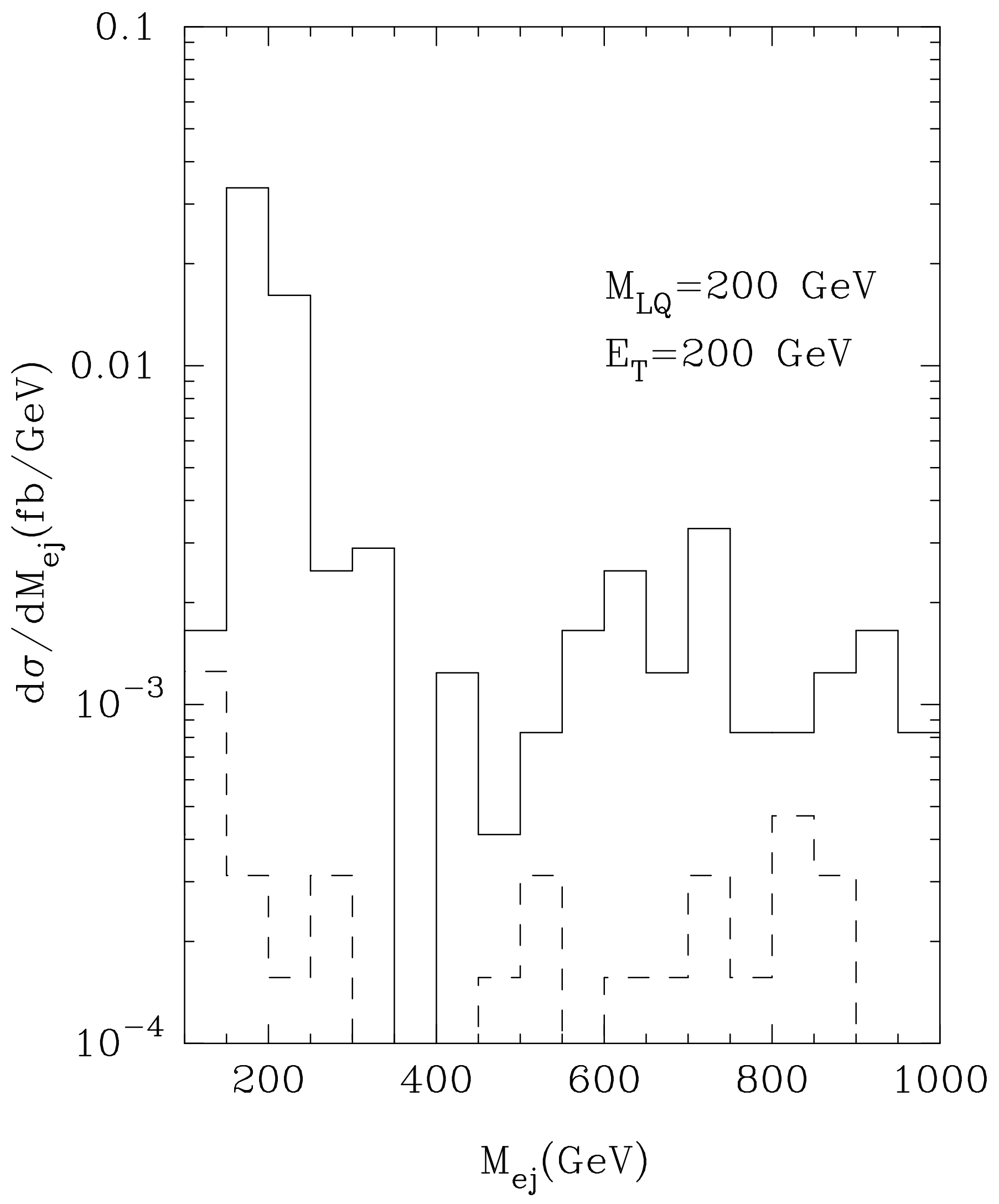


Figure 5b

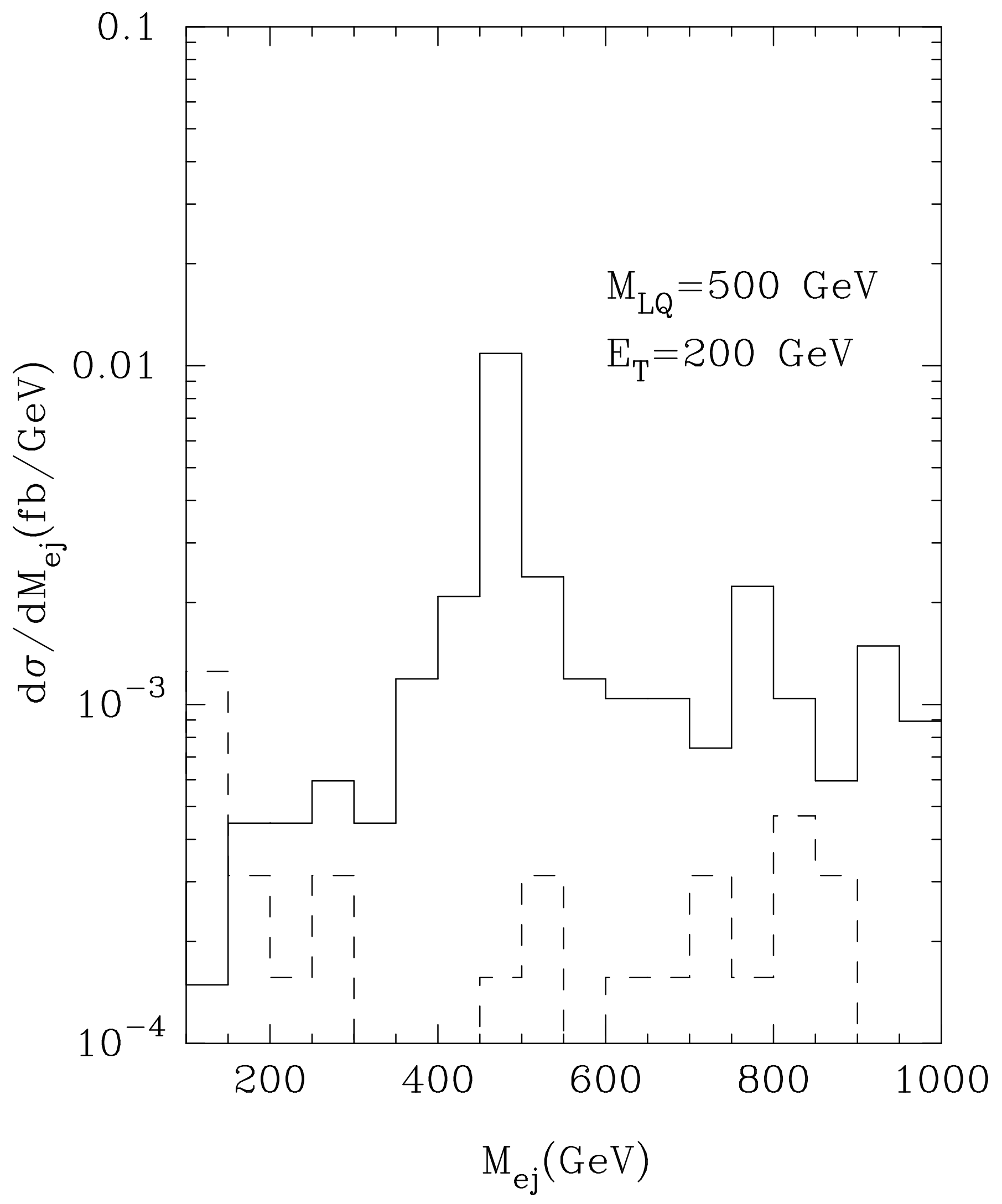


Figure 5c

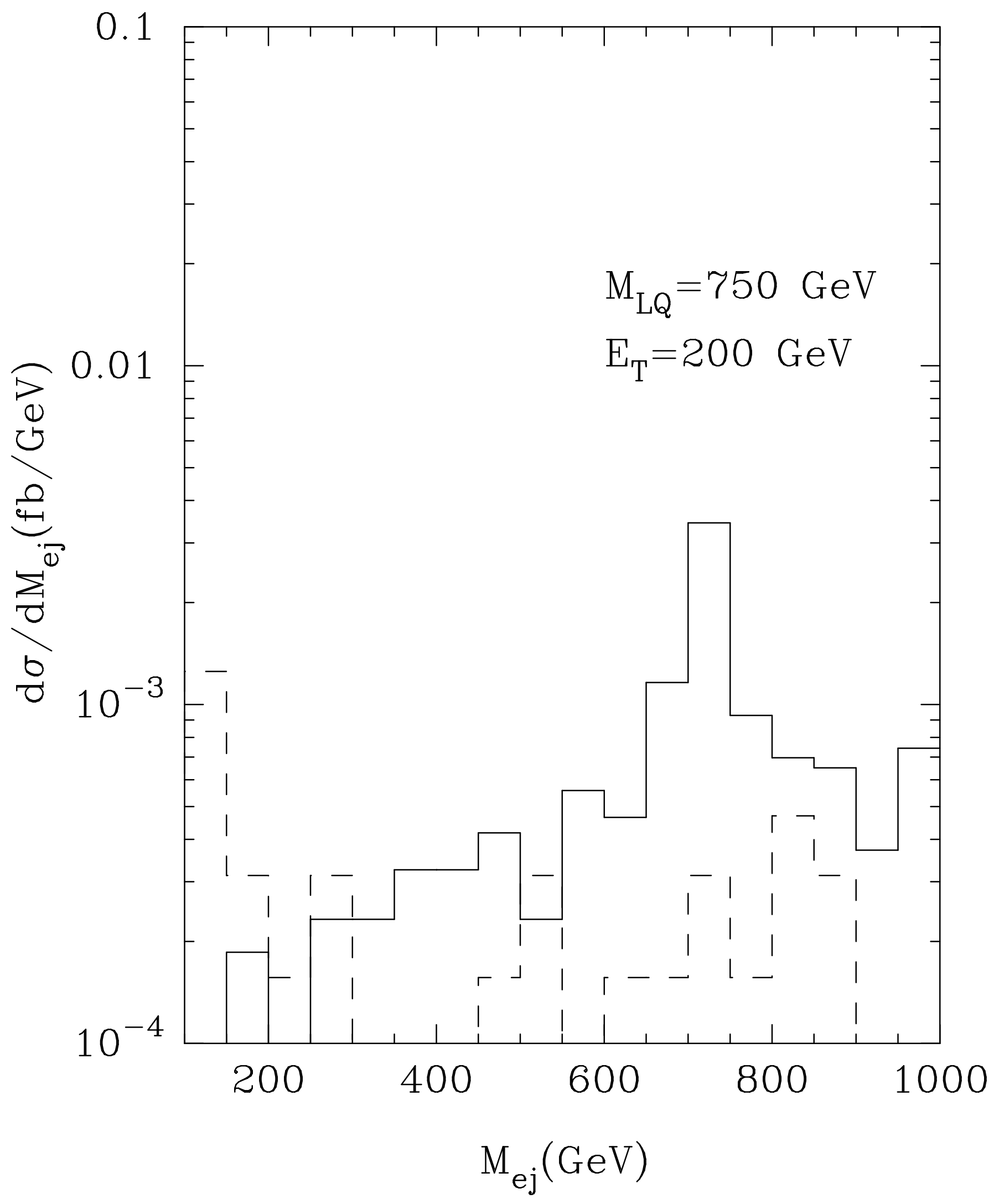


Figure 6

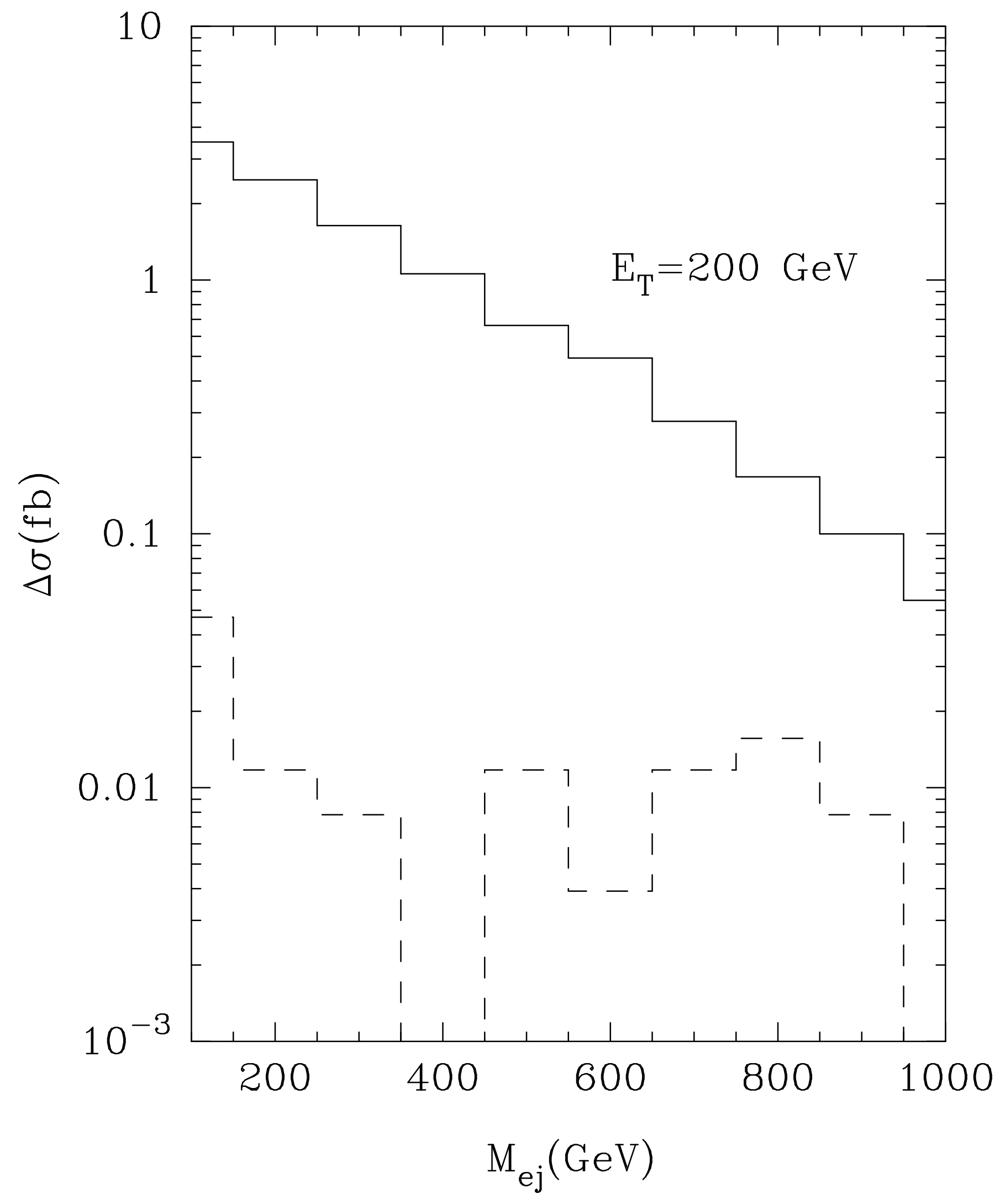

